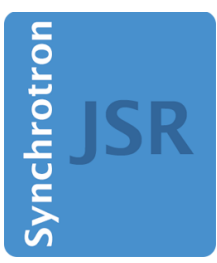

JOURNAL OF

SYNCHROTRON

RADIATION

Volume 23 (2016)

Supporting information for article:

Non-Negative Matrix Factorization for the near real time interpretation of absorption effects in elemental distribution images acquired by XRF imaging

Matthias Alfeld, Mirwaes Wahabzada, Christian Bauckhage, Kristian Kersting, Gerd Wellenreuther, Pere Barriobero-Vila, Guillermo Requena, Ulrike Boesenberg and Gerald Falkenberg 


\subsection{Signal gradients at interphase}
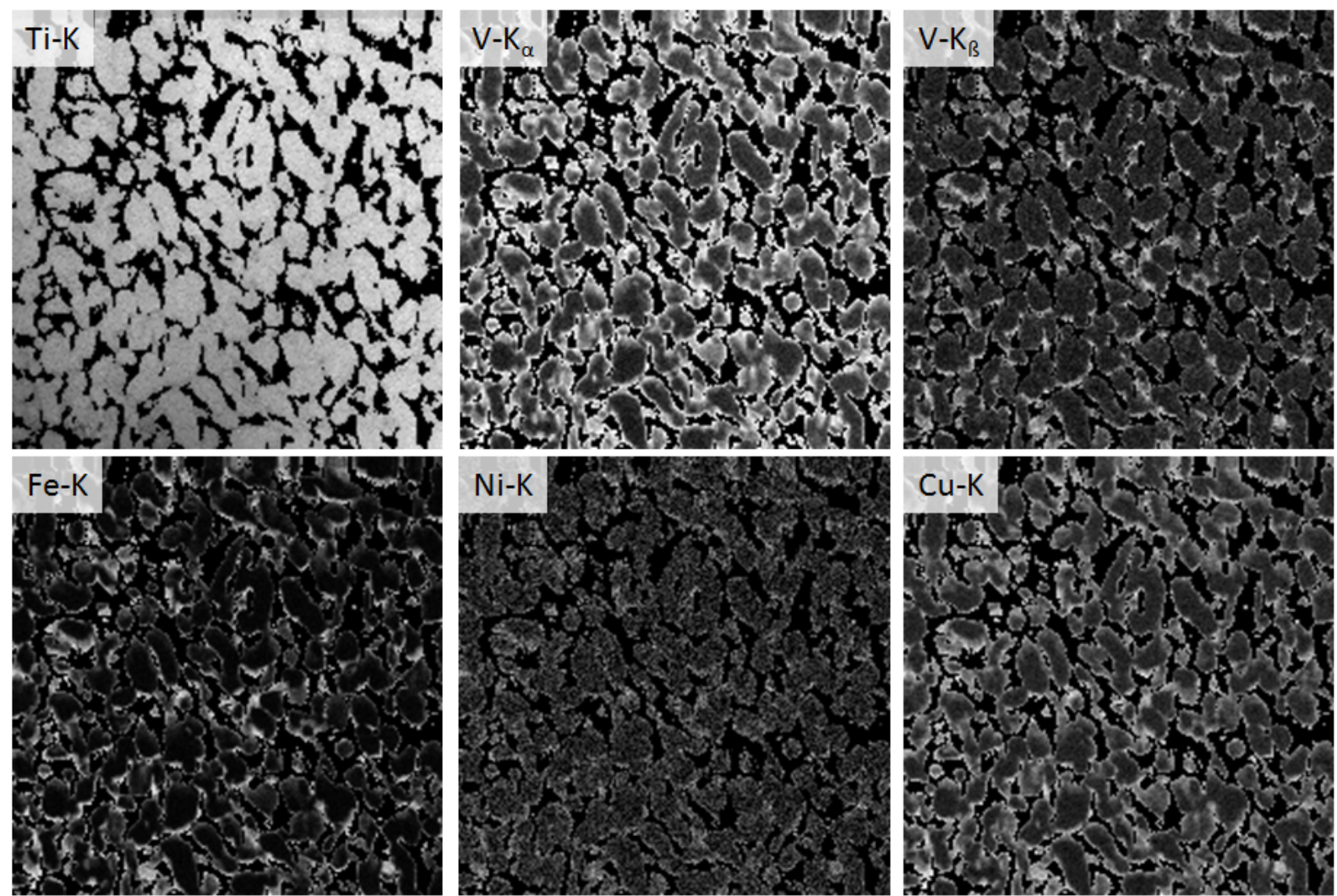

Figure-DSI 1 Elemental distribution images of sample B. In all images the pixels associated with the $\beta$-phase, based on a threshold set in the $\mathrm{V}-\mathrm{K}_{\alpha}$ image, have been rendered black.

In Figure-DSI 1 the same elemental distribution images of sample B are shown as in Figure 2 of the main text. To enhance the visibility of the signal gradient at the interphase between primary $\alpha$ and $\beta$ phases the pixels attributed to the $\beta$-phase are rendered black. The differentiation between primary $\alpha$ and $\beta$ phases was done, based on a threshold set on the $V-K_{\alpha}$ image. The signal gradient is clearly discernible in the $\mathrm{V}-\mathrm{K}_{\alpha}$, Fe-K and $\mathrm{Cu}-\mathrm{K}$ images and to a lesser degree in the $\mathrm{V}-\mathrm{K}_{\beta}$ and $\mathrm{Ni}-\mathrm{K}$ images. In the Ti-K image no significant decrease at the borders of the selected area can be observed, which confirms that the threshold has not been set too restrictive. 


\subsection{NMF with 4 bases}

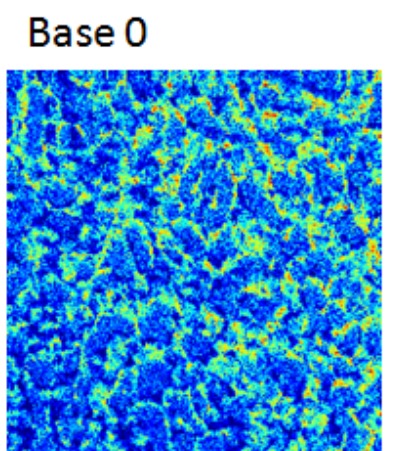

Base 1

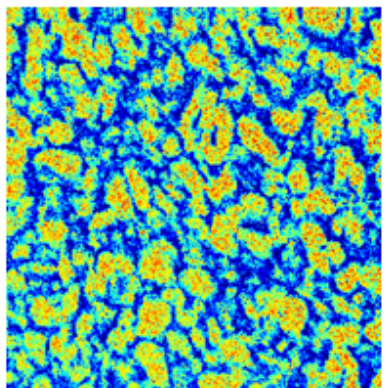

Base 2

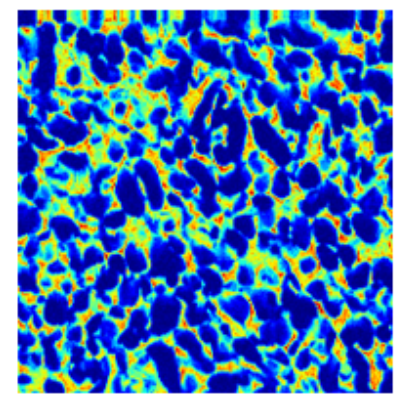

1000

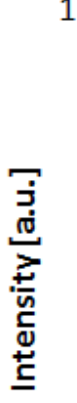

Base vectors

100

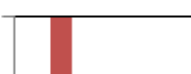

normalized to $\mathrm{V}-\mathrm{Ka}$

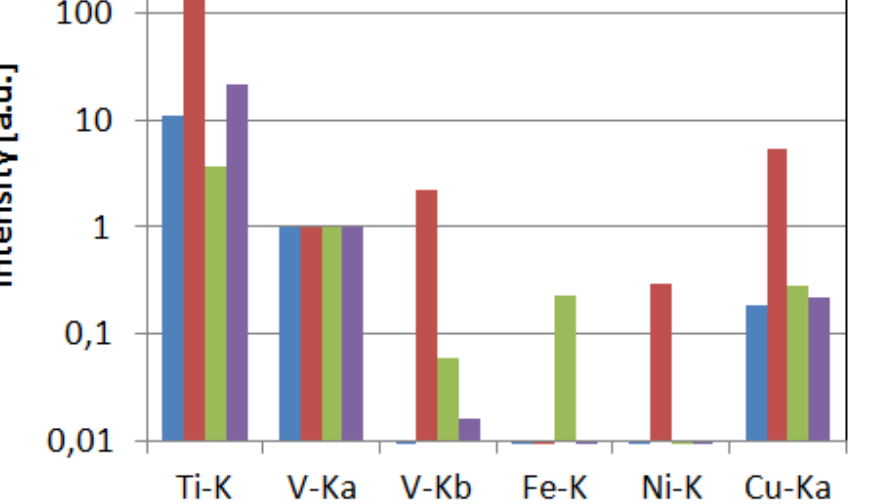

Base 3

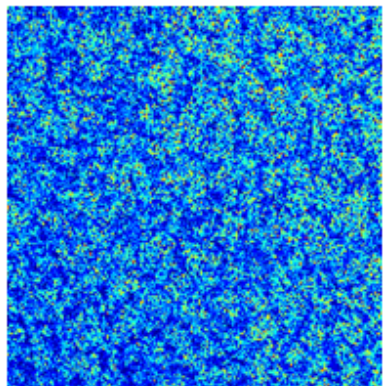

Base 0

Base 1

Base 2

Base 3

Figure-DSI 2 NMF of sample B. The same settings as in main text were used, but the number of bases was enhanced from 3 to 4.

The NMF results of sample B shown in Figure-DSI 2 were calculated with the same setting as those shown in Figure 3 and 4 of the main text, but with four bases $(k=4)$ instead of three $(k=3)$. The coefficient images shown here are more noisy, but the first three are in general in good agreement with their counterparts in the main text. The fourth base, Base 3 , contains mainly signals from the Tirich $\alpha$ phase.

The loads on the base vectors of Base 0 and Base 2 are comparable to those obtained with $\mathrm{k}=3$ bases, but the values for Base 1 have changed, as the Ti-rich $\alpha$ phase is now represented by the sum of the Bases 1 and 3. 


\subsection{Failure of convergence}

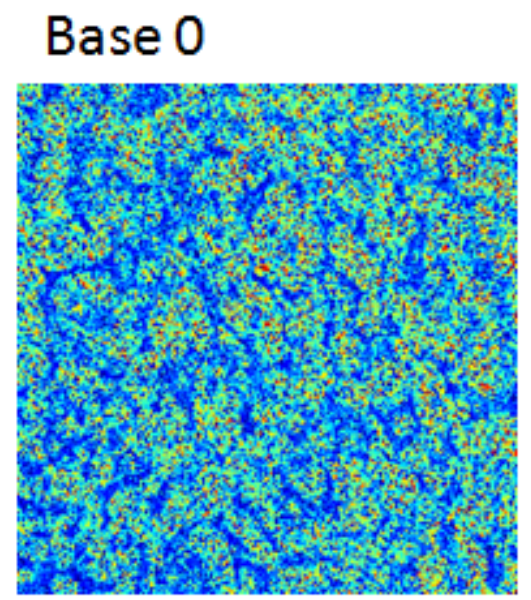

Base 1

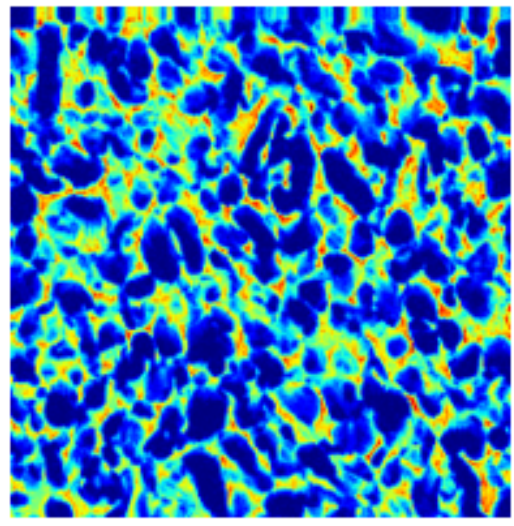

Base 2

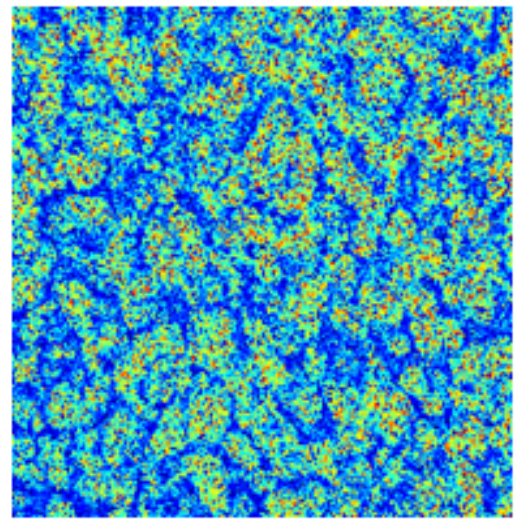

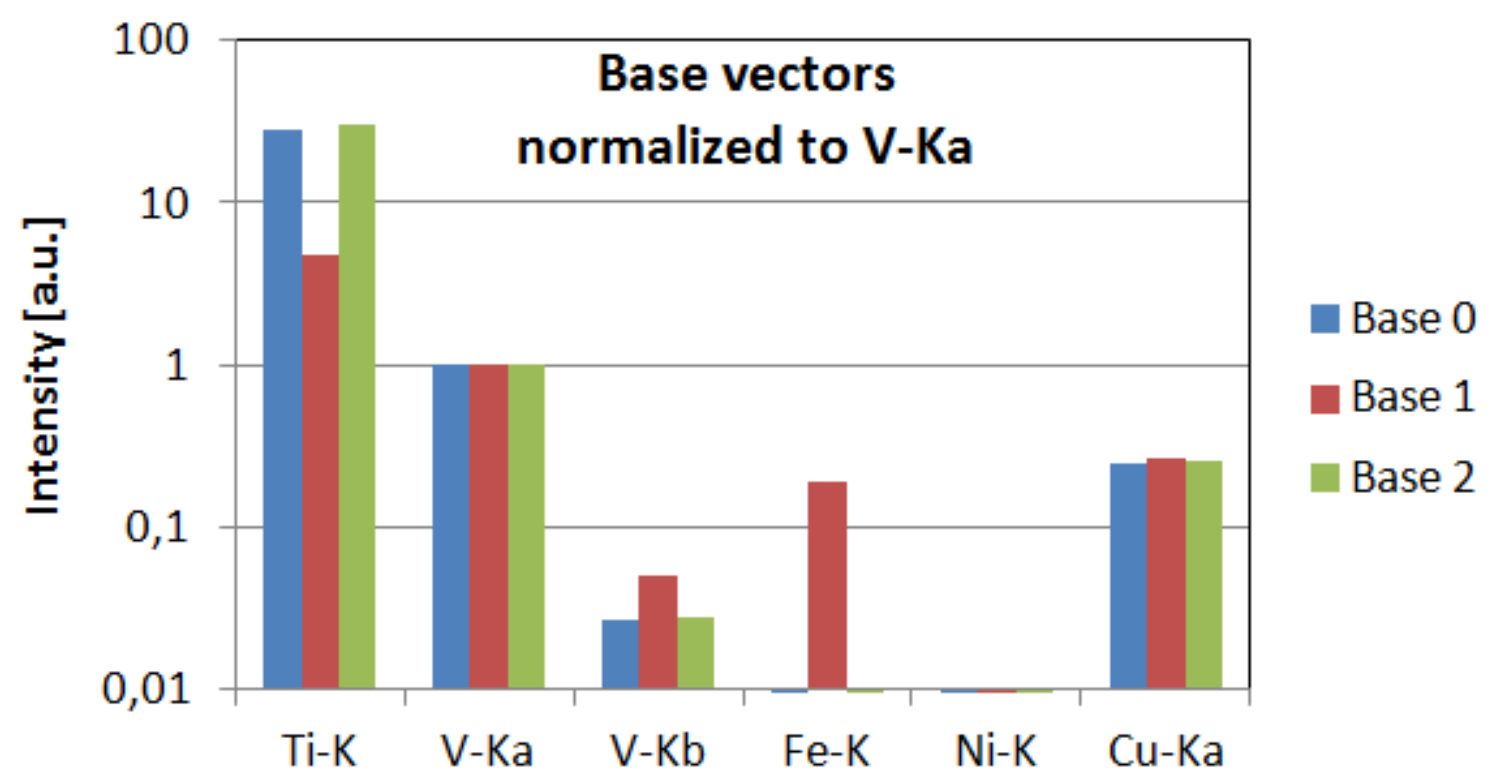

Figure-DSI 3 NMF of sample B, which failed due to "wrong" initial random values, resulting in an improper representation. Same settings as in main text.

With some random initial values for $\mathrm{W}$ and $\mathrm{H}$ the representation can be early trapped in a local minimum of the response function. In the case shown in Figure-DSI 3 Base 0 and Base 2 represent the Ti-rich $\alpha$ phase, while Base 1 represents the $V$-rich $\beta$ phase. From this representation the anisotropic features resulting from absorption effects in the border between $\alpha$ and $\beta$ phase are not apparent. 


\subsection{Misguiding results}

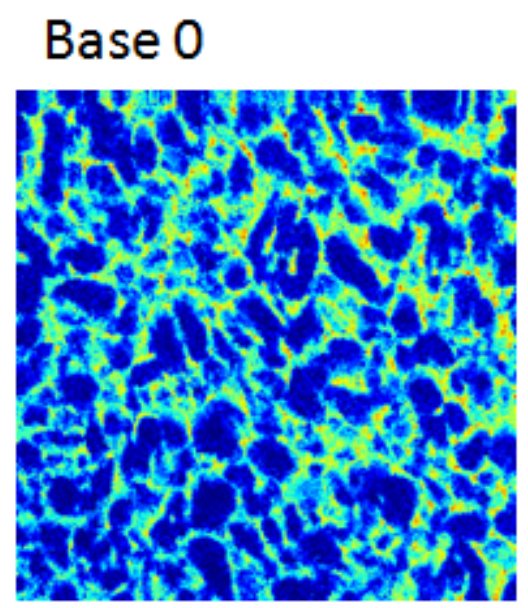

Base 1

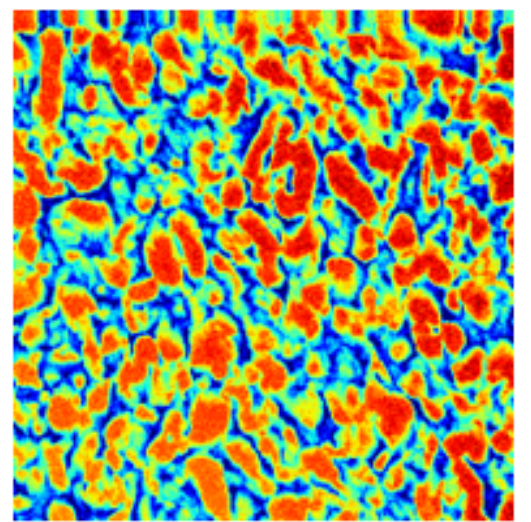

Base 2

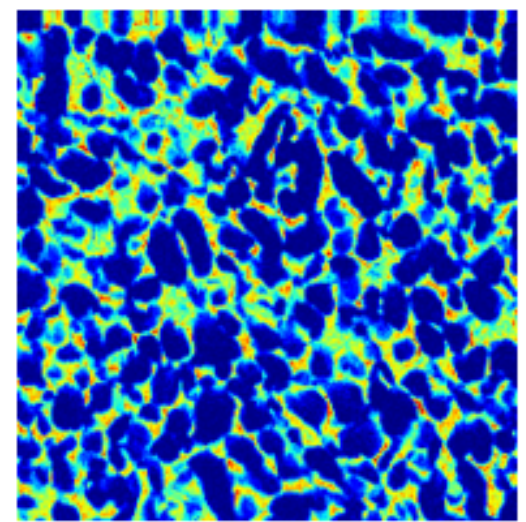

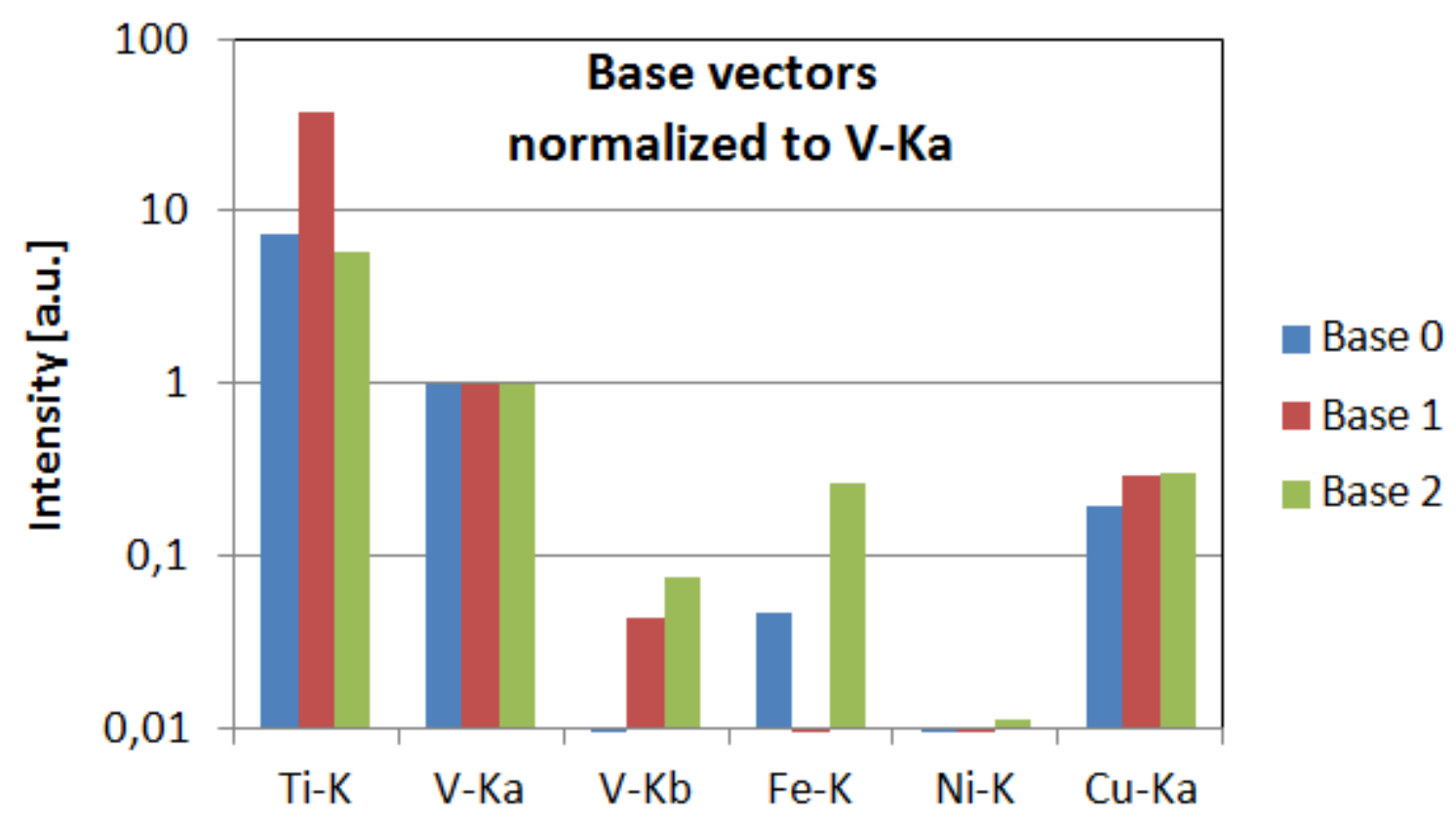

Figure-DSI 4 NMF of sample B, which failed due to "wrong" initial random values, resulting in an improper and misguiding representation. Same settings as in main text.

In Figure-DSI 4 a misguiding representation of the data set of sample $B$ is shown. Base 1 represents the Ti-rich $\alpha$ phase, while Base 2 represents the $V$-rich $\beta$ phase. However, the areas of the $\alpha$ phase are more pronounced in the coefficient map of Base 1 than in the "successful" representation shown in the main text. Base 0 still represents the anisotropic absorption effects in the phase borders, but these are less obvious than the in the main text. The loads on the bases also differ significantly. The results shown here are a correct representation of the data set of sample $B$, but considerably more difficult to be interpreted. 


\subsection{Principal Component Analysis (PCA)}

\section{Base 0}

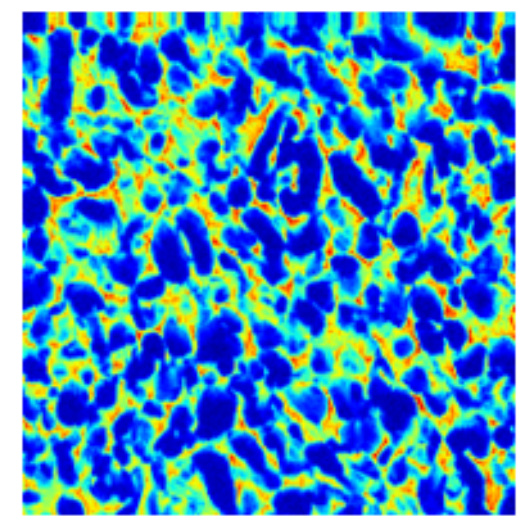

Base 1

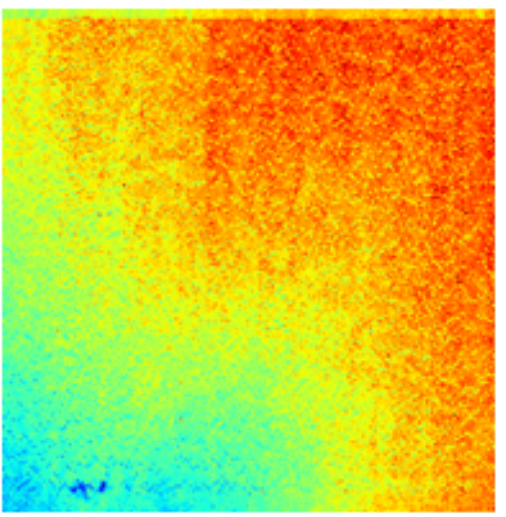

Base 2

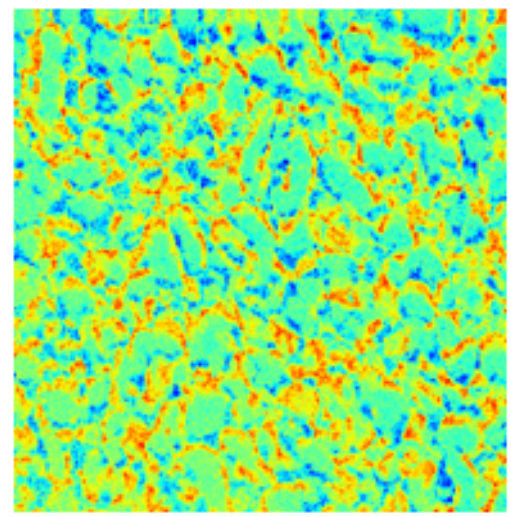

PCA loads - Normalized to V-Ka

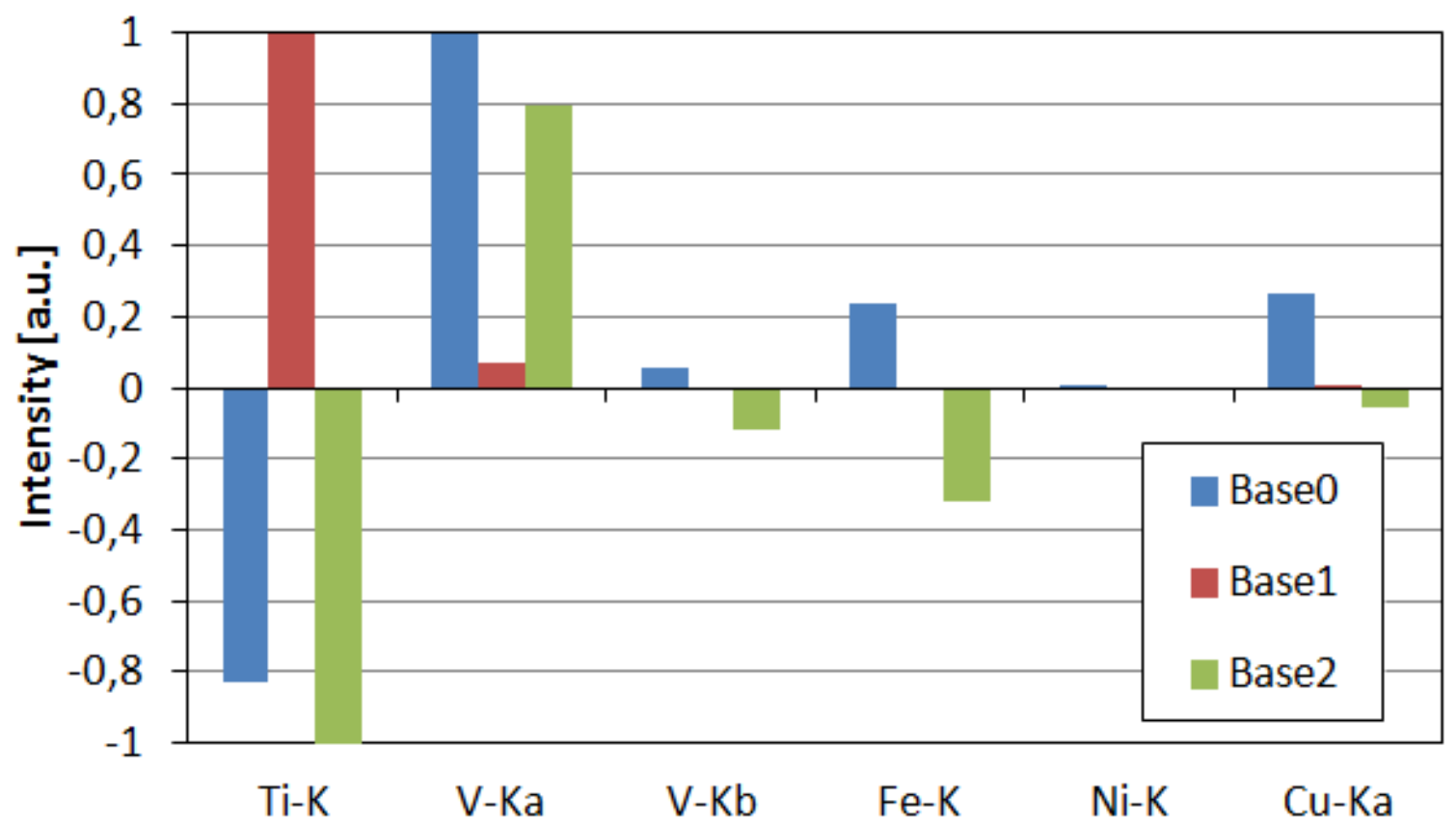

Figure-DSI 5 Principal component Analysis of sample B.

In Figure-DSI 5 the PCA of sample B is shown. The data was normalized as for the NMF. The coefficient image of Base 0 is similar to the distribution of the $\beta$-phase. Its loads consequently show a notable anti-correlation of Ti with $\mathrm{V}$ and the minor compounds stabilizing the $\beta$-phase. Base 1 features for all elements positive loads and represents the variation of sensitivity, due to thickness variation of the sample. This effect is strongest for the only weakly absorbed Ti-K radiation. Base 2 highlights the anisotropic absorption effects in a similar way to the Base 0 of the successful NMF discussed in the main text. Also the anti-correlation of $\mathrm{V}-\mathrm{K}_{\alpha}$ to all other signals is clearly shown.

It is noteworthy that the results by PCA were obtained in less than a second, faster than NMF, and the results are not dependent on random initial values. The PCA Base 1 also provides information not directly available by NMF under the chosen settings. However, the partly negative nature of the loads renders the interpretation more difficult. It is easy to interpret PCA base 2 in combination with 
the $\mathrm{MC}$ simulation in the main text, but without a priori knowledge this can be considered challenging.

\subsection{Scatter plot}
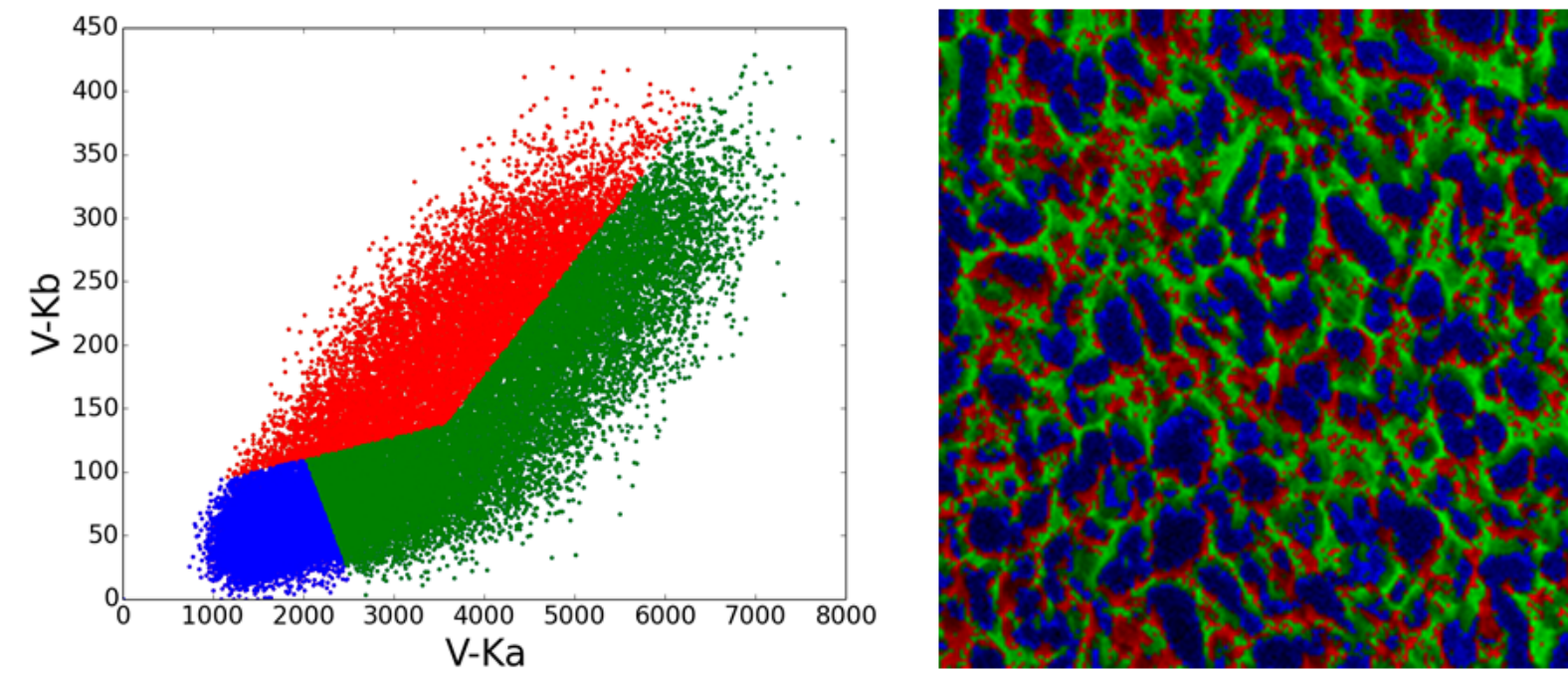

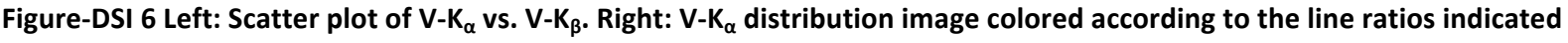
in the scatter plot.

It holds, in general, true that the ratio between fluorescence lines resulting from the same inner shell vacancy is rather constant. Here this is not valid due to the presence of an absorption edge between them. In the left of Figure-DSI 6 the signals of $V-K_{\alpha}$ is plotted against that of $V-K_{\beta}$ and a broadening of the distribution can be observed. One can color an elemental distribution image according to clusters manually selected in the scatter plot, as shown on the right of Figure-DSI 6 . The blue cluster corresponds to the Ti-rich $\alpha$ phase, while the green cluster represents the $V$-rich $\beta$ phase. In the red cluster the signal of $\mathrm{V}-\mathrm{K}_{\beta}$ is enhanced to that of $\mathrm{V}-\mathrm{K}_{\alpha}$; these are regions in which the $\beta$ phase is on the surface of the sample. It is worth noting that here regions are highlighted that appear dark in Base 0 of Figure 3 and 4 of the main text.

While this approach provides easily interpretable results and allows the direct use of a priori knowledge, its disadvantages are that it requires a high degree of user intervention to select the clusters and it is also limited to the simultaneous investigation of two elements. 


\subsection{Line-ratio map}
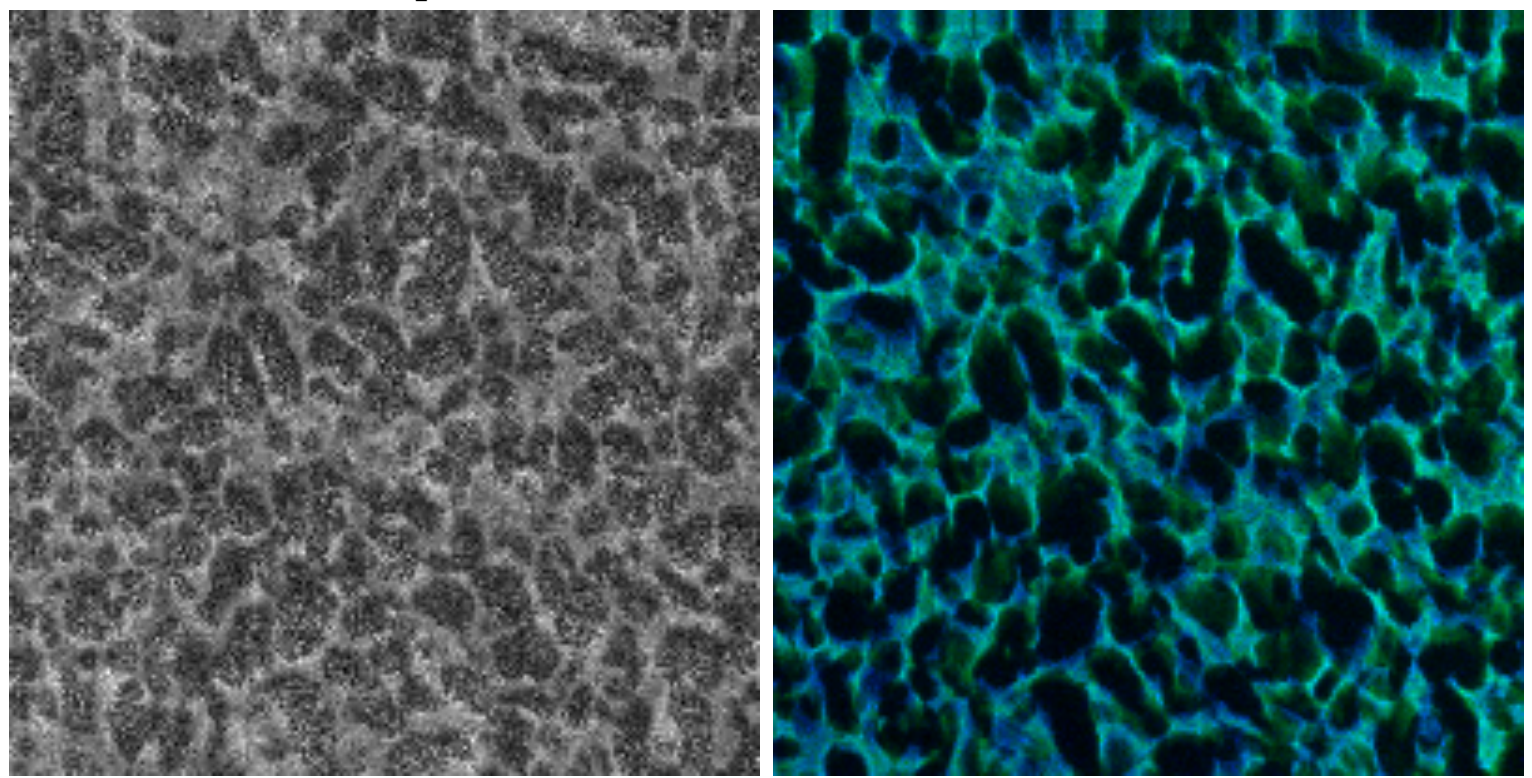

Figure-DSI 7 Left: Map of the $V-K_{\beta} /\left(V-K_{\beta}+V-K_{\alpha}\right)$ ratio. Right: Two color image: $V-K_{\alpha}$ (green) and $V-K_{\beta}(b l u e)$.

Instead of studying the ratio of elements in a scatter plot, one can also investigate them directly in the images. The first approach shown is a simple ratio map in the form of $\mathrm{V}-\mathrm{K}_{\beta} /\left(\mathrm{V}-\mathrm{K}_{\beta}+\mathrm{V}-\mathrm{K}_{\alpha}\right)$. This form of map was chosen, as a direct ratio map (i.e. $V-K_{\beta} / V-K_{\alpha}$ ) is prone to produce extreme values in pixels with bad statistics. The ratio map shown in the left of Figure-DSI 7 reveals, if compared with elemental distribution images of $\mathrm{V}$ or $\mathrm{Ti}$, that the line ratio of $\mathrm{V}-\mathrm{K}$ is anisotropic in the borders between $\alpha$ and $\beta$ phase due to the absorption effects. This, however, requires a close inspection of the images, which makes it impractical to use this approach for the study of more than a few line ratios.

In the right of Figure-DSI 7 a two color image is shown, in which $\mathrm{V}-\mathrm{K}_{\alpha}$ is used in the green channel and $\mathrm{V}-\mathrm{K}_{\beta}$ in the blue one. It is clearly discernible that in the (dark) $\alpha$ grains a variable amount of $\mathrm{V}$ is present, which is in a different ratio to the $V-K_{\beta}$ signal than in the $\beta$ phase. While the image is less noisy and allows for the inspection of up to three elements in parallel, it still requires a high level of direct user intervention to be interpreted. 